\title{
福建省两栖类物种多样性评估
}

\author{
耿宝荣* \\ （福建师范大学生物工程学院 福州 350007)
}

\begin{abstract}
摘要: 地区性物种多样性评价是当前全球生物多样性研究的重要组成部分。本文采用 G-F 指数对福建省两栖类物 种多样性进行评估, 同时利用弦距离类间平均连锁的方法对福建省 22 个县 (市) 和 5 个两栖动物地理省进行聚类 分析。结果表明: 福建省各县 (市) 的两栖类物种多样性差异较大。其中武夷山市两栖类的 $G$ 指数达 2.465 , 为各 县最高; 闽中地理省两栖类的 $\mathrm{G}$ 指数达 2.534 , 为各两栖动物地理省最高; 全省两栖类物种多样性的 $\mathrm{G}$ 指数、 $\mathrm{F}$ 指数 和 G-F 指数分别为 2.978 、 4.863 和 0.388 。处于华中区的 3 个地理省 (闽北、闽东和闽西地理省) 首先聚类, 而后与 处于华南区的闽中地理省聚类, 最后与闽南地理省合并。聚类结果与福建省两栖动物地理区划大体一致, 但个别 县(市) 不太相符, 作者建议将大田县划归在华中区的闽西地理省。

关键词: G-F 指数, 聚类分析, 生物多样性, 两栖动物, 福建省
\end{abstract}

中图分类号：Q958.122 文献标识码: A 文章编号: 1005-0094(2004)06-0618-08

\section{Evaluation of amphibian species diversity in Fujian}

GENG Bao-Rong*

Bioengineering College, Fujian Normal University, Fuzhou 350007

\begin{abstract}
Evaluation of regional species diversity is an important component in studying global biodiversity. In this paper, amphibian species diversity ( ASD) in Fujian Province was evaluated using the G-F index and cluster analysis with chord distance ( CRD) to measure between-groups linkages. The analysis was conducted on the amphibian compositions in 22 county or city areas and five zoogeographic divisions. Differences between counties or cities were significant. For the G-index of ASD, Wuyishan scored the highest value (2.465) among the counties or cities; and central Fujian zoogeographic division scored 2.534, the highest value of the five divisions. The G-index, F-index and G-F index of the entire Fujian ASD are 2.978, 4. 863 and 0.388 , respectively. The cluster analysis based on CRD showed that the northern, eastern, and western Fujian zoogeographic divisions, located in Central China Zoogeographic Region, formed a group, which successively clustered with the central and the southern zoogeographic divisions in the South China Zoogeographic Region. The linkage pattern based on counties or cities is similar to that based on zoogeographic divisions. The author suggests that Datian County, though located in central Fujian, should be placed in the western Fujian zoogeographic division.
\end{abstract}

Key words: G-F index, cluster analysis, biodiversity, amphibians, Fujian Province

近年来全球环境恶化日趋严重,生物多样性研 究越来越受到科学家的关注, 世界各地学者都将保 护当地生物多样性当作重要的研究课题 (李培芬 等,2000)。物种是生物分类系统的基本单元, 物种 多样性是生物多样性的基本层次, 研究、测度物种多 样性对于保护生物多样性具有重要意义( Wilson, 1988 )。为了提高生物多样性研究与保护的水平和 可靠性, 为物种多样性的长期监测和保护等提供坚
实的科学基础, 寻求物种多样性评估的科学途径与 方法具有重要价值 (周红章, 2000)。我国学者蒋志 刚和纪力强 (1999) 提出了基于信息测度的 G-F 指 数, 用于客观地评估地区性物种多样性。

福建省东临太平洋, 土地面积 12.14 万 $\mathrm{km}^{2}$, 以 低山丘陵为主, 森林覆盖率位居全国第一位; 常绿阔 叶林是福建省的地带性植被主体 (朱锦禁等, 2003 )。福建省地处亚热带, 属温暖湿润的亚热带 
海洋性季风气候, 由于武夷山脉和鹑峰山-戴云山博平岭形成的特殊地形以及东南季风的影响, 具有 丰富的水热资源和两栖动物资源。福建省共有两栖 动物 46 种 (耿宝荣, 2002), 隶属于 2 目 9 科 24 属 (费梁, 1999; 费梁等, 2001,2002), 但各地区及各两 栖动物地理省的多样性差异较大。本文利用 G-F 指数及弦距离聚类分析方法对福建省两栖动物分布 进行评估与相关分析，以期能够较为客观地对区域 性两栖动物多样性进行评估, 并为三级动物地理区 划提供依据。

\section{1 研究方法}

以福建省境内两栖动物分布数据为研究对象, 其中分布数据包括两个不同区域类型( 行政县市和 两栖动物地理省) 的两栖动物名录。资料来自作者 近年的野外调查, 并结合前人调查结果 (蔡明章, 1995; 费梁, 1999; 费梁和叶昌媛, 1992; 费梁等, 2001,2002; 耿宝荣,2002; 耿宝荣和蔡明章, 1995; 江 建平和周开亚, 2001; 李成等, 2001; Pope, 1931; 丁 汉波等, 1980; 叶昌媛和费梁, 1994,1995)。从中选 择调查较彻底全面、记载较完整以及在正式学术刊 物上有报道的 22 个县(市) (图 1 ) 的 41 种两栖动 物, 分别建立各县(市) 和各两栖动物地理省的数据 矩阵。

行政区划按现行的行政区划划分; 两栖动物地 理省区划按《福建省两栖动物地理区划》一文 (耿宝 荣,2002) 划分。

多样性评估采用 G-F 指数 (蒋志刚和纪力强, 1999):

(1) $\mathrm{F}$ 指数 $\left(D_{F}\right)$ : 在一个特定的科 $k, D_{F k}=$ $-\sum_{i=1}^{n} p_{i} \ln p_{i}$, 其中: $p_{i}=s_{k i} / S_{k}, S_{k}=$ 福建省两栖动物名 录(下简称名录) 中科 $k$ 中的物种数, $s_{k i}=$ 名录中科 $k$ 属 $i$ 中的物种数, $n=$ 科 $k$ 中的属数。一个地区的 $\mathrm{F}$ 指数 $D_{F}=\sum_{k=1}^{m} D_{F k}$, 其中: $m=$ 名录中两栖纲的科数。

(2) G 指数 $\left(D_{G}\right): D_{G}=-\sum_{j=1}^{p} q_{j} \ln q_{j}$, 其中: $q_{j}=s_{j}$ / $S, S=$ 名录中两栖纲的物种数, $s_{j}=$ 两栖纲中属 $j$ 中 的物种数, $p=$ 两栖纲中的属数。

\section{(3) G-F 指数: $D_{G-F}=1-D_{G} / D_{F}$}

聚类分析采用弦距离 ( chord distance, CRD) 类 间平均连锁对策 ( between-groups linkage) 的方法, 分

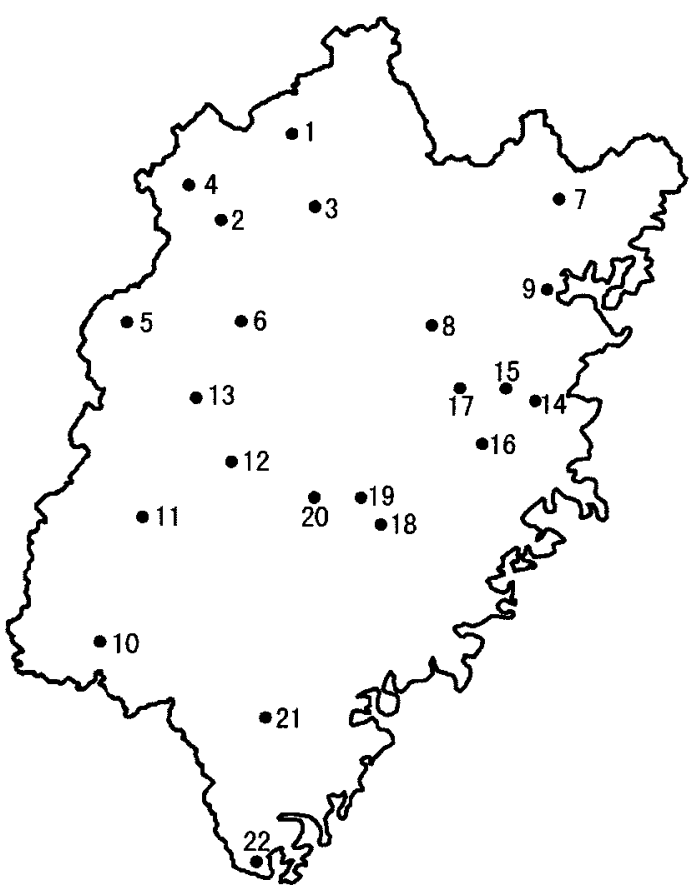

图 1 用于分析福建省两栖类物种多样性的 $\mathbf{2 2}$ 个县(市) 的 分布

1 : 武夷山; 2 : 邵武; 3 : 建阳; 4 : 光泽; 5 : 建宁; 6 : 将乐; 7 : 福 安; 8: 古田; 9: 罗源； 10 : 上杭；11: 连城； 12 : 永安； 13 : 明 溪; 14: 福州; 15 : 闽侯; 16 : 永泰; 17 : 闽清; 18 : 永春; 19 : 德 化; 20 : 大田; 21 : 南靖; 22 : 诏安

Fig. 1 Distribution of counties and cities used to analyse amphibian species diversity in Fujian Province

1, Wuyishan; 2, Shaowu; 3, Jianyang; 4, Guangze; 5, Jianning; 6, Jiangle; 7, Fuan; 8, Gutian; 9, Luoyuan; 10, Shanghang; 11, Liancheng; 12, Yongan; 13, Mingxi; 14, Fuzhou; 15, Minhou; 16, Yongtai; 17, Minqing; 18, Yongchun; 19, Dehua; 20, Datian; 21, Nanjing; 22, Zhaoan

别建立福建省各县(市) 和各两栖动物地理省的 $0 、 1$ 分布表 (表 1 ), 以距离系数作相似性指标, 用系统聚 类法分析其相似性, 得出福建省 22 县 (市) 和 5 个 两栖动物地理省的聚类图。数据处理采用 SPSS 11.0软件进行。

\section{2 结果与分析}

2.1 福建省 22 个县 (市) 和 5 个两栖动物地理省 的 G-F 指数

按 G-F 指数公式分别计算出福建省 22 个县 (市) 和 5 个两栖动物地理省的两栖类物种多样性 的 $\mathrm{F}$ 指数 $\left(D_{F}\right) 、 \mathrm{G}$ 指数 $\left(D_{G}\right)$ 和 G-F 指数 $\left(D_{G-F}\right)$ (表 $2,3)$ 。

从表 2 看出, 22 个县 (市) 的两栖动物分布物种 
表 1 福建省两栖动物的种类与分布

Table 1 Distribution of amphibian species in Fujian Province

福建两栖动物地理省

物种

Amphibian zoogeographic divisions of Fujian Province

Species

闽东 East

$\left(\mathrm{VA}_{\mathrm{F} 2}\right)$

闽西 West

闽中 Central

$\left(\mathrm{VII} \mathrm{A}_{\mathrm{F} 1}\right)$

闽南 South

$\left(\mathrm{VA}_{\mathrm{F} 1}\right.$ )

$\left(\mathrm{VA}_{\mathrm{F} 3}\right)$

0

0

大鲵 Andrias davidianus

1

1

0

蝶螈科 Salamandridae

东方蝾螈 Cynops orientalis

黑斑肥螈 Pachytriton brevipes

中国㾮螈 Paramesotriton chinensis

锄足蟾科 Pelobatidae

福建掌突蟾 Leptolalax liui

淡肩角蟾 Megophrys boettgeri

挂墩角蟾 $M$. kuatunensis

刘氏髼蟾 Vibrissaphora liui

蟾蜍科 Bufonidae

中华蟾蜍Bufo gargarizans

黑睈蟾蜍 B. melanostictus

树蟾科 Hylidae

中国树蟾 Hyla chinensis

三港树蟾 H. sanchiangensis

蛙科 Ranidae

崇安湍蛙 Amolops chunganensis

戴云湍蛙 A. daiyunensis

华南湍蛙 A. ricketti

武夷湍蛙 A. wuyiensis

泽陆蛙 Fejervarya multistriata

小腺蛙 Glandirana minima

虎纹蛙 Hoplobatrachus rugulosus

弹琴水蛙 Hylarana (Hylarana) adenopleura

沼水蛙 H. (Hylarana) guentheri

阔褶水蛙 H. (Hylarana) latouchii

台北纤蛙 H. (Tenuirana) taipehensis

福建大头蛙 Limnonectes fujianensis

尖舌浮蛙 Occidozyga lima

竹叶臭蛙 Odorrana exiliversabilis

大绿臭蛙 $O$. livida

花臭蛙 $O$. schmackeri

小棘蛙 Paa exilispinosa

九龙棘蛙 $P$. jiulongensis

棘胸蛙 P. spinosa

黑斑侧褶蛙 Pelophylax nigromaculata

金线侧褶蛙福建亚种 P. plancyi fukienensis

镇海林蛙 Rana zhenhaiensis

树蛙科 Rhacophoridae

大树蛙 Rhacophorus dennysi

斑腿树蛙 R. megacephalus

姬蛙科 Microhylidae

花狭口蛙 Kaloula pulchra

粗皮姬蛙 Microhyla butleri

小弧斑姬蛙 $M$. heymonsi

饰纹姬蛙 $M$. ornata

花姬蛙 $M$. . pulchra
1

0

1
1
1

1

1

1

1

1

0

0

1

1
1

1
1
0

$\begin{array}{ll}1 & 1 \\ 1 & 1 \\ 1 & 0\end{array}$

$\begin{array}{ll}1 & 1 \\ 1 & 1 \\ 1 & 0 \\ 1 & 0\end{array}$


表 2 福建省 22 个县市两栖类物种多样性的 G-F 指数

Table 2 G-F index of amphibian species in different areas of Fujian Province

\begin{tabular}{lcccc}
\hline \multicolumn{1}{c}{$\begin{array}{c}\text { 县(市) } \\
\text { Counties } \\
\text { or cities }\end{array}$} & $\begin{array}{c}\text { 物种数 } \\
\text { Number } \\
\text { of species }\end{array}$ & $\begin{array}{c}\text { G 指数 } \\
\text { G-index }\end{array}$ & $\begin{array}{c}\text { F 指数 } \\
\text { F-index }\end{array}$ & $\begin{array}{c}\text { G-F 指数 } \\
\text { G-F index }\end{array}$ \\
\hline 武夷山 Wuyishan & 33 & 2.465 & 4.005 & 0.385 \\
邵武 Shaowu & 26 & 2.038 & 3.767 & 0.459 \\
建阳 Jianyang & 29 & 2.229 & 4.245 & 0.475 \\
光泽 Guangze & 28 & 2.216 & 4.221 & 0.475 \\
建宁 Jianning & 27 & 2.069 & 3.761 & 0.450 \\
将乐 Jiangle & 25 & 2.038 & 3.767 & 0.459 \\
福安 Fuan & 27 & 2.162 & 4.133 & 0.477 \\
古田 Gutian & 26 & 2.072 & 4.133 & 0.499 \\
罗源 Luoyuan & 26 & 2.072 & 4.133 & 0.499 \\
上杭 Shanghang & 30 & 2.210 & 3.695 & 0.402 \\
连城 Liancheng & 30 & 2.265 & 3.823 & 0.407 \\
永安 Yongan & 25 & 1.981 & 3.767 & 0.474 \\
明溪 Mingxi & 25 & 2.038 & 3.767 & 0.459 \\
福州 Fuzhou & 28 & 2.310 & 4.492 & 0.486 \\
闽侯 Minhou & 27 & 2.162 & 4.274 & 0.494 \\
永泰 Yongtai & 26 & 2.137 & 4.459 & 0.521 \\
闽清 Minqing & 30 & 2.260 & 4.258 & 0.469 \\
永春 Yongchun & 24 & 1.878 & 3.489 & 0.462 \\
德化 Dehua & 31 & 2.454 & 4.042 & 0.393 \\
大田 Datian & 26 & 1.979 & 3.414 & 0.420 \\
南靖 Nanjing & 26 & 1.983 & 3.680 & 0.461 \\
诏安 Zhaoan & 27 & 2.095 & 4.110 & 0.490 \\
平均 Average & & 2.142 & 3.974 & 0.460 \\
\hline & & & & \\
\hline
\end{tabular}

数最多的是武夷山市, 达 33 种; 其代表属间的多样 性指数 $\left(D_{G}=2.465\right)$ 为各县 (市) 最高; 而相应地, 其 代表科间多样性指数 $\left(D_{F}=4.005\right)$ 仅略高于平均值 (3.974), 而 G-F 指数 $\left(D_{G-F}=0.385\right)$ 为各县 (市) 的 最低值。永泰县的 G-F 指数 $\left(D_{G-F}\right)$ 达到 0.521 , 为 各县(市)最高, 主要是由于其科间多样性指数高所 致。从 5 个两栖动物地理省的情况看, 闽中两栖动 物地理省 $\left(V I I A_{\mathrm{F} 1}\right)$ 的种类最多, 其 $\mathrm{G}$ 指数最高 $\left(D_{G}=\right.$ $2.534)$; 闽东两栖动物地理省 $\left(\mathrm{VI}_{\mathrm{F} 2}\right)$ 的 $\mathrm{G}$ 指数最 低 $\left(D_{G}=2.162\right.$ ) (表 3 )。

福建省总的属间多样性指数 ( $\mathrm{G}$ 指数) 为 2.978 , 科间多样性指数 ( $F$ 指数) 为 4.863 , 均高于 各县及各动物地理省的指数 (表 3 )。因为县 (市) 或动物地理省为行政省次一级的区划, 种类数及科 属数较少。

2.2 福建省 22 个县( 市) 和 5 个两栖动物地理省 的聚类分析

以 3 种距离与 4 种对策构成的 12 种聚类方法 进行分析, 从中篮选出最合适的弦距离 (CRD) (表
4、5) 类间平均连锁对策 ( between-groups linkage) 的 聚类方法, 分别作福建省 22 个县市和 5 个两栖动物 地理省的聚类图( 图 2、3)。

从图 2 可知,22 个县(市) 中两栖动物分布无差 异的是古田县和罗源县, 永安市、明溪县和将乐县, 其弦距离为 0 。第 20 次聚类时弦距离最大, 其次是 第 16 次, 它们是跳跃点。取第 16 次聚类的中间结 合点 $[\mathrm{CRD}=(0.132+0.105) / 2=0.1185]$ 为阈值, 将 22 个县(市) 分为 6 类: 闽南的南靖县和诏安县 为一类; 闽西的上杭县、连城县和大田县为第二类; 闽北、闽东及福州地区的县 (市) 为第三类; 闽中地 区的永泰、永春和德化三县各为一类。取第 20 次聚 类的中间结合点 $[\mathrm{CRD}=(0.225+0.170) / 2=$ 0.1975 ]为阈值时, 22 个县市可分为两类, 闽南地区 的南靖县、诏安县为一类, 其余各县 (市) 合为一类。 南靖县、诏安县位于福建南部, 含有福建省所有的 4 种东洋界华南区种类，而缺少由北向南分布的古北 界种类, 与其他各县 (市) 形成明显差别, 单聚为一 类与实际情况相符。

从图 3 可见, 5 个两栖动物地理省通过四次聚 类并在一起。其中第三次的弦距离 ( 以中间结合点 为阈值, CRD 为 0.1725 ) 最大, 第二次 (以中间结合 点为阈值, CRD 为 0.1255 ) 次之。在弦距离为 0.086 时, 闽北地理省与闽东地理省先聚为一类; 当 弦距离为 0.1255 时聚为 3 类, 即属华中区的 3 个地 理省( 闽北地理省、闽东地理省和闽西地理省) 归为 一类,华南区的 2 个地理省 (闽中地理省和闽南地 理省) 各一类。当弦距离为 0.1725 时, 闽中地理省 与华中区的地理省聚在一起, 而闽南地理省则单为 一类。由于闽中地理省位于福建中部和戴云山脉的 东部，向北分布的东洋界华南区种类和向南分布的 华中区种类在此地区交错,一定程度上影响了华南 区特征的体现,所以形成了这种先与华中区聚类,而 后才与闽南地理省聚类的结果。

\section{3 讨论}

生物多样性指数是物种丰富度与均匀度结合起 来的一个单一统计量 (马克平和刘玉明, 1994), 而 G-F 指数是基于物种数目的一种快速、有效的测度 物种多样性的方法( 蒋志刚和纪力强, 1999)。G-指 数用于测度一个地区的属间物种多样性, F-指数测 度的是科中和科间多样性，二者所测定的生物分类 
表 3 福建省 5 个两栖动物地理省的 G-F 指数

Table 3 G-F index of five amphibian zoogeographic divisions in Fujian Province

\begin{tabular}{ccccc}
\hline $\begin{array}{c}\text { 动物地理省 } \\
\text { Zoogeographic divisions }\end{array}$ & $\begin{array}{c}\text { 物种数 } \\
\text { Number of species }\end{array}$ & $\begin{array}{c}\text { G 指数 } \\
\text { G-index }\end{array}$ & $\begin{array}{c}\text { F 指数 } \\
\text { F-index }\end{array}$ & $\begin{array}{c}\text { G-F 指数 } \\
\text { G-F index }\end{array}$ \\
\hline$V I A_{\mathrm{F} 1}$ & 34 & 2.509 & 3.945 & 0.364 \\
$V \mathrm{~F}_{\mathrm{F} 2}$ & 27 & 2.162 & 4.133 & 0.477 \\
$V \mathrm{VA} \mathrm{F}_{\mathrm{F} 3}$ & 32 & 2.345 & 3.748 & 0.374 \\
$V I A_{\mathrm{F} 1}$ & 37 & 2.534 & 4.465 & 0.432 \\
$V I I A_{\mathrm{F} 2}$ & 29 & 2.368 & 4.426 & 0.465 \\
福建省 Fujian Province & 41 & 2.978 & 4.863 & 0.388 \\
\hline
\end{tabular}

$\mathrm{VI}_{\mathrm{F} 1}-\mathrm{VII} A_{\mathrm{F} 2}$ 代表的地理省同表 $1 \quad \mathrm{VIA}_{\mathrm{F} 1}-\mathrm{VII} \mathrm{A}_{\mathrm{F} 2}$ correspond to those in Table 1

表 4 福建省 22 个县(市) 间两栖动物相同物种数和分布弦距离 *

Table 4 The common species and CRD of distribution of amphibians among 22 counties or cities in Fujian Province*

\begin{tabular}{|c|c|c|c|c|c|c|c|c|c|c|c|c|c|c|c|c|c|c|c|c|c|c|}
\hline & $1^{* *}$ & 2 & 3 & 4 & 5 & 6 & 7 & 8 & 9 & 10 & 11 & 12 & 13 & 14 & 15 & 16 & 17 & 18 & 19 & 20 & 21 & 22 \\
\hline 1 & & 26 & 29 & 28 & 26 & 25 & 26 & 25 & 25 & 26 & 27 & 25 & 25 & 25 & 25 & 23 & 28 & 22 & 27 & 24 & 21 & 22 \\
\hline 2 & 0.112 & & 26 & 26 & 24 & 25 & 25 & 25 & 25 & 25 & 25 & 25 & 25 & 25 & 25 & 22 & 25 & 22 & 24 & 23 & 20 & 21 \\
\hline 4 & 0.079 & 0.036 & 0.017 & & 25 & 25 & 25 & 25 & 25 & 25 & 25 & 25 & 25 & 25 & 25 & 22 & 26 & 22 & 25 & 23 & 20 & 21 \\
\hline 5 & 0.129 & 0.094 & 0.107 & 0.091 & & 24 & 24 & 24 & 24 & 24 & 24 & 24 & 24 & 24 & 24 & 22 & 26 & 21 & 24 & 24 & 21 & 19 \\
\hline 6 & 0.130 & 0.019 & 0.072 & 0.055 & 0.076 & & 25 & 25 & 25 & 24 & 24 & 25 & 25 & 25 & 25 & 21 & 25 & 22 & 23 & 23 & 20 & 20 \\
\hline 8 & 0.147 & 0.038 & 0.090 & 0.073 & 0.094 & 0.019 & 0.019 & & 26 & 24 & 24 & 25 & 25 & 26 & 26 & 22 & 26 & 22 & 23 & 23 & 20 & 20 \\
\hline 9 & 0.147 & 0.038 & 0.090 & 0.073 & 0.094 & 0.019 & 0.019 & 0.000 & & 24 & 24 & 25 & 25 & 26 & 26 & 22 & 26 & 22 & 23 & 23 & 20 & 20 \\
\hline 10 & 0.174 & 0.105 & 0.119 & 0.137 & 0.157 & 0.124 & 0.157 & 0.141 & 0.141 & & 29 & 24 & 24 & 24 & 24 & 22 & 26 & 23 & 26 & 25 & 25 & 25 \\
\hline 11 & 0.142 & 0.105 & 0.119 & 0.137 & 0.157 & 0.124 & 0.122 & 0.141 & 0.141 & 0.033 & & 24 & 24 & 24 & 24 & 22 & 26 & 22 & 26 & 25 & 24 & 24 \\
\hline 12 & 0.130 & 0.019 & 0.072 & 0.055 & 0.076 & 0.000 & 0.038 & 0.019 & 0.019 & 0.124 & 0.124 & & 25 & 25 & 25 & 21 & 25 & 22 & 23 & 23 & 20 & 20 \\
\hline 16 & 0.215 & 0.154 & 0.199 & 0.185 & 0.170 & 0.176 & 0.170 & 0.154 & 0.154 & 0.212 & 0.212 & 0.1760 & 0.176 & 0.148 & 0.132 & & 24 & 21 & 24 & 21 & 19 & 19 \\
\hline 17 & 0.110 & 0.105 & 0.119 & 0.137 & 0.086 & 0.087 & 0.086 & 0.069 & 0.069 & 0.133 & 0.133 & 0.0870 & 0.087 & 0.103 & 0.0860 & .141 & & 22 & 25 & 25 & 22 & 21 \\
\hline 18 & 0.218 & 0.119 & 0.166 & 0.151 & 0.175 & 0.102 & 0.136 & 0.119 & 0.119 & 0.143 & 0.180 & 0.1020 & 0.102 & 0.113 & 0.0960 & .1590 & ). 180 & & 22 & 20 & 20 & 20 \\
\hline 19 & 0.156 & 0.155 & 0.133 & 0.151 & 0.170 & 0.174 & 0.205 & 0.190 & 0.190 & 0.147 & 0.1470 & 0.1740 & 0.174 & $0.151 \mathrm{C}$ & 0.1700 & .1550 & 0.180 & 0.193 & & 25 & 22 & 22 \\
\hline 20 & 0.181 & 0.115 & 0.126 & 0.148 & 0.094 & 0.098 & 0.132 & 0.115 & 0.115 & 0.105 & 0.105 & 0.0980 & 0.098 & 0.148 & 0.1320 & ). 1920 & . 105 & 0.1990 & 0.119 & & 22 & 20 \\
\hline 21 & 0.283 & 0.231 & 0.235 & 0.259 & 0.207 & 0.216 & 0.245 & 0.231 & 0.231 & 0.105 & 0.141 & 0.2160 & 0.216 & 0.222 & 0.2450 & ). 2690 & .212 & 0.1990 & 0.225 & .154 & & 24 \\
\hline 22 & 0.263 & 0.207 & 0.214 & 0.236 & 0.2960 & 0.230 & 0.2590 & 0.245 & 0.245 & 0.122 & 0.1570 & 0.2300 & 0.230 & 0.2360 & 0.2590 & .2830 & 0.262 & 0.214 & 0.240 & 0.245 & 0.094 & \\
\hline
\end{tabular}

* 对角线右上方数字表示各县(市)的共有种数,左下方的数字表示二者的余弦距离 The figures above diagonal are the common species shared by horizontal and vertical counties or cities, and the figures below diagonal are the CRD of them.

** 1-22 为各县(市) 的代码 (见图 1) Numbers 1-22 indicate different counties or cities (see Fig. 1)

表 5 福建省 5 个两栖动物地理省间相同种数和分布弦距离

Table 5 The common species and CRD among five amphibian zoogeographic divisions in Fujian Province

\begin{tabular}{cccccc}
\hline $\begin{array}{c}\text { 动物地理区划 } \\
\text { Zoogeographic divisions }\end{array}$ & $\mathrm{VIA}_{\mathrm{F} 1}$ & $\mathrm{VIA}_{\mathrm{F} 2}$ & $\mathrm{VIA}_{\mathrm{F} 3}$ & $\mathrm{VII}_{\mathrm{F} 1}$ & $\mathrm{VII}_{\mathrm{F} 2}$ \\
\hline $\mathrm{VIA}_{\mathrm{F} 1}$ & & 26 & 28 & 29 & 22 \\
$\mathrm{VI} \mathrm{F}_{\mathrm{F} 2}$ & 0.086 & & 26 & 26 & 21 \\
$\mathrm{VIA}_{\mathrm{F} 3}$ & 0.096 & 0.115 & & 30 & 26 \\
$\mathrm{VII}_{\mathrm{F} 1}$ & 0.130 & 0.177 & 0.128 & & 26 \\
$\mathrm{VII}_{\mathrm{F} 2}$ & 0.241 & 0.236 & 0.131 & 0.192 & \\
\hline
\end{tabular}

对角线右上方数字表示各地理省的共有种数,对应左下方的数字表示二者的余弦距离 The figures above diagonal are common species shared by horizontal and vertical amphibian zoogeographic divisions, and the figures below diagonal are the CRD of them. 


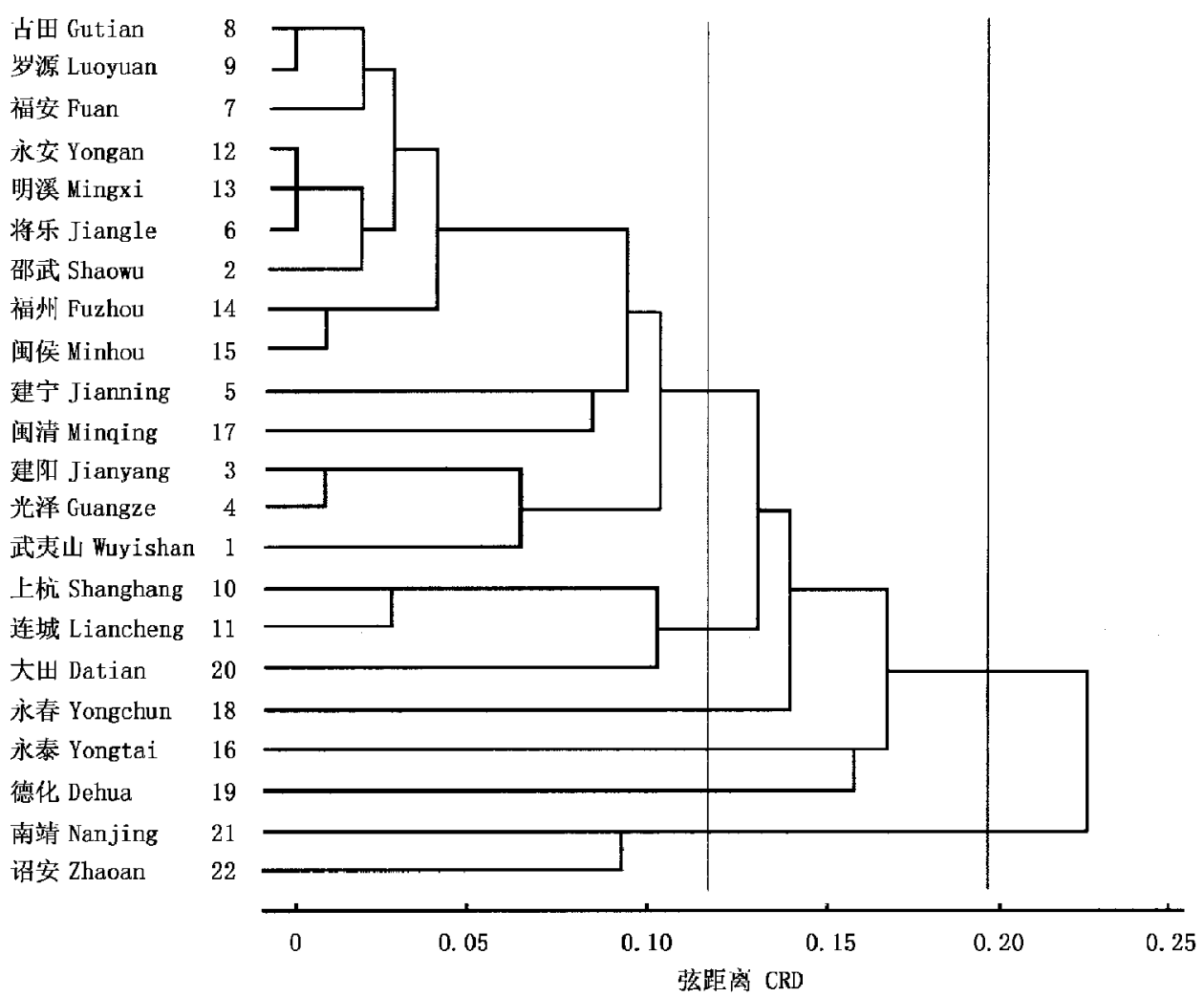

图 2 福建省 22 个县(市)两栖动物分布的弦距离聚类树

Fig. 2 Cluster analysis of amphibian distribution in 22 counties or cities in Fujian Province based on CRD

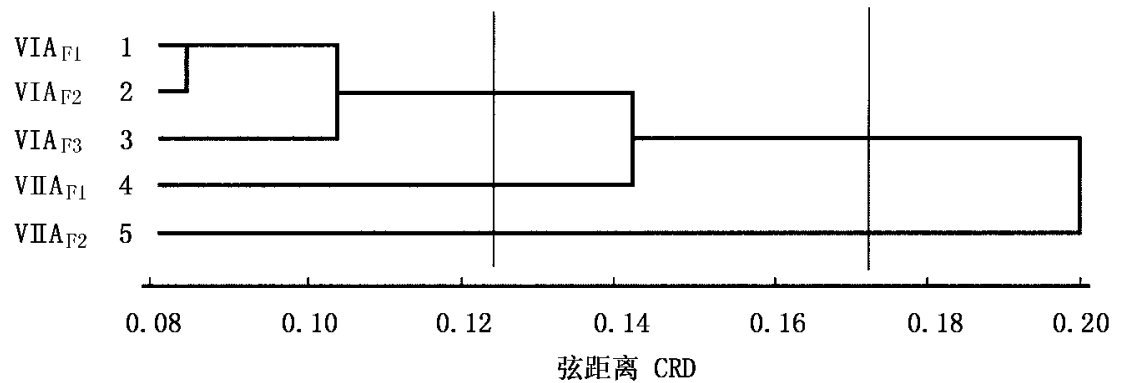

图 3 福建省 5 个两栖动物地理省的弦距离聚类树 ( $V \mathrm{VA}_{\mathrm{F} 1}-\mathrm{VII} \mathrm{A}_{\mathrm{F} 2}$ 代表的地理省同表 1)

Fig. 3 Cluster analysis of five amphibian zoogeographic divisions in Fujian Province based on CRD. VI $A_{\mathrm{F} 1}-V_{I I} A_{\mathrm{F} 2}$ correspond to those in Table 1

层次不同。G-F 指数是一种标准化指数, 可进行不 同地区间生物多样性比较。蒋志刚和纪力强 (1999)运用该指数对我国 9 个地区的鸟类和兽类 进行了物种多样性的评估。本文的研究表明, 该指 数同样可用于地区性的两栖类物种多样性的测度。

在福建省 22 个县(市)中,武夷山市的两栖类 $G$ 指数最高; 在 5 个两栖动物地理省中, 闽中地理省的 $\mathrm{G}$ 指数最高。这种现象与自然地理条件有一定的关
系。武夷山市地处武夷山脉北段, 环境独特, 地势 高, 山谷溪涧错综分布, 植被类型多样化, 适合于两 栖动物的生存与繁衍; 闽中地理省位于福建省中部 东面, 温暖湿润, 雨量充沛, 西面多为中低山地, 东部 为福州小平原, 许多华中区和华南区种类在本区域 分布和互相渗透, 形成了福建省两栖类物种多样性 最丰富的一个地理省。由此可见, G-F 指数, 尤其是 $\mathrm{G}$ 指数在很大程度上能体现出地区性两栖类物种多 
样性的程度。

地区间物种丰富度和均匀度的差异将导致 G-F 指数的差异。在福建省, 许多县 (市) 的两栖类物种 数是相等的 (如永泰县和南靖县, 建宁县和闽侯县) (表 2), 但由于它们的均匀度不同 (物种分布于各 属、科的情况不同), 反映在 G-F 指数上是有差别 的。

两栖动物在福建省分布的聚类分析, 与福建省 两栖动物地理区划 (耿宝荣, 2002) 基本一致。大多 地理位置相靠近的县 (市) 都先聚类, 然后再与地理 位置较远的县(市) 聚类。闽北、闽东和闽西两栖动 物地理省位于华中区, 在聚类分析上先聚为一类, 而 后才与位于华南区的闽中和闽南地理省聚类。

少数县(市)的聚类结果与地理区划不一致, 可 能是与该地区两栖动物资源的调查不够详尽有关, 亦有可能是以前的区划存在一定的局限性。从本研 究看, 大田县处于闽中地理省和闽西地理省的交界 处, 但聚类分析结果中偏向于闽西地理省, 与以前区 划 ( 耿宝荣, 2002) 不一致, 作者建议将大田县划归 在闽西两栖动物省 $\left(\mathrm{VIA}_{\mathrm{F} 3}\right)$, 即将华中区与华南区 在福建省的中部分界线向东移动约 $0.2^{\circ}$ 。闽中地 理省与闽南地理省位于华南区, 在聚类分析上并非 先聚为一类,而是闽中地理省先与华中区的 3 个地 理省的合并项聚类, 而后再与闽南地理省聚类, 这与 两栖动物地理区划 (耿宝荣, 2002) 有一定差异。作 者认为, 这是由于闽中地理省处于福建省境内的华 中区与华南区的交界处,华中区和华南区的许多物 种在本区域重叠分布, 不能充分体现华南区的特征 所造成的。

丁汉波等(1980) 曾用两栖动物的区系成分特 征对福建省的两栖动物地理进行了区划, 将福建省 两栖动物划分为 5 个动物地理区系, 为福建省两栖 动物的三级地理区划提供了有益资料。但文章仅从 较大的地区和直观的成分比例来划分, 对较小区域 (如县) 的区划和归属则难以确定; 同时无法体现不 同分类阶元 (如科、属) 层次上的差异。本文对小地 域的县(市) 和大地域的地区两方面同时进行研究, 并以标准化的 G-F 指数及弦距离聚类的分析方法 进行研究, 一定程度上可以弥补之不足。

\section{参考文献}

Cai, M. Z. (蔡明章). 1995. A survey of amphibian of the
Wuyishan Nature Reserve with an analysis of their regional faunistic composition. Journal of Fujian Normal University (Natural Science) [ 福建师范大学学报(自然科学版)], 11: 82 - 85. (in Chinese with English abstract)

Fei, L. (费梁). 1999. Atlas of Amphibians of China (中国两栖 动物图鉴). Henan Science and Technology Press, Zhengzhou. (in Chinese)

Fei, L. (费梁) and Ye, C. Y. (叶昌媛). 1992. Comments on the taxonomy of pelobatid toads of genus Leptolalax (Carpophrys) with description of a new species. Acta Zoologica Sinica (动物学报), 38: 245 - 253. (in Chinese with English abstract)

Fei, L. (费梁), Ye, C. Y. (叶昌媛), Jiang, J. P. (江建平) and Xie, F. (谢锋). 2002. On taxonomic status of Rana limnocharis group with revision of nomenclature of the rice frog from China. Herpetologica Sinica (两栖爬行动物学研 究), 9:88-96. (in Chinese with English abstract)

Fei, L. (费梁), Ye, C. Y. (叶昌媛) and Li, C. (李成). 2001. Taxonomic studies of Odorrana versabilis in China. II. Descriptions of two new species (Amphibia: Ranidae). Acta Zootaxonomica Sinica (动物分类学报), 26: 601-607. (in Chinese with English abstract)

Geng, B. R. (耿宝荣). 2002. Amphibian fauna and zoogeographic division of Fujian. Sichuan Journal of Zoology ( 四 川动物) , 21: $170-174$. (in Chinese)

Geng, B. R. (耿宝荣) and Cai, M. Z. (蔡明章). 1995. An investigation and fauna comparison of amphibian in Zhaoan, Yongtai and Jianning counties of Fujian Province. Journal of Fujian Normal University ( Natural Science) [ 福建师范大 学学报(自然科学版)], 11: $78-81$. (in Chinese with English abstract)

Jiang, J. P. (汇建平) and Zhou, K. Y. (周开亚). 2001. Evolutionary relationships among Chinese ranid frogs inferred from mitochondrial DNA sequences of 12S rRNA gene. Acta Zoologica Sinica (动物学报), 47: 38 - 44. (in Chinese with English abstract)

Jiang, Z. G. (蒋志刚) and Ji, L. Q. (纪力强). 1999. Avianmammalian species diversity in nine representative sites in China. Chinese Biodiversity (生物多样性), 7:220 - 225 . (in Chinese with English abstract)

Lee, P. F. (李培芬), Pan, Y. H. (潘彦宏), Lü, G. Y. (吕光 洋), Chou, W. H. (周文豪) and Chang, C. R. (张琪如). 2000. Distribution models and biodiversity of amphibians in Taiwan. In: Organizational Committee of Seminar of CrossStraits Biodiversity and Conservation (ed. ), Proceedings of Seminar of Cross-Straits Biodiversity and Conservation (海峡 两岸生物多样性与保育研讨会论文集). National Museum of Natural Science, Taizhong, 159 - 177. (in Chinese with English abstract) 
Li, C. (李成), Ye, C. Y. (叶昌媛) and Fei, L. (费梁). 2001. Taxonomic studies of Odorrana versabilis in China. I. Taxonomic status of the geographic populations (Amphibia: Ranidae). Acta Zootaxonomica Sinica (动物分类学报), 26: 593 - 600. ( in Chinese with English abstract)

Ma, K. P. (马克平) and Liu, Y. M. (刘玉明). 1994. Measurement of biotic community diversity. I. $\alpha$ diversity. Chinese Biodiversity (生物多样性), 2:231 - 239. (in Chinese)

Pope, C. H. 1931. Notes on amphibians from Fujian, Hainan and other parts of China. Bulletin of the American Museum of Natural History, 58: $334-487$.

Ting, H. P. ( 丁汉波)，Zheng, J. (郑辑) and Cai, M. C. (蔡明 章). 1980. A study on the geographical distribution and regional faunistic composition of the Amphibia and Reptilia of Fujian Province. Journal of Fujian Normal University (Natural Science) [福建师范大学学报(自然科学版)], (1) : 57 - 74. (in Chinese with English abstract)

Wilson, E. O. 1988. Biodiversity. National Academy Press, Washington, D. C.

Ye, C. Y. (叶昌媛) and Fei, L. (费梁). 1994. A new species of family Ranidae-Limnonectes fujianensis from Fujian, China (Amphibia: Anura). Acta Zootaxonomica Sinica (动 物分类学报), 19: 494 - 499. (in Chinese with English abstract)

Ye, C. Y. (叶昌媛) and Fei, L. (费梁) . 1995. Taxonomic studies on the small type Megophrys in China including descriptions of the new species ( subspecies) (Pelobatidae: genus Megophrys). Acta Herpetologica Sinica (两栖爬行动物学 研究), (4/5): 72 -81. (in Chinese with English abstract) Zhou, H. Z. (周红章). 2000. Species and species diversity. Chinese Biodiversity (生物多样性), 8: 215 - 226. (in Chinese with English abstract)

Zhu, J. M. (朱锦禁), Chen, Y. Q. (陈由强) and Huang, R. Z. (黄儒珠). 2003. Distribution pattern of nature reserves in Fujian and biodiversity reserve. In: Fujian Association for Science and Technology et al. (eds.), Proceedings of the First Forum of Cross-Straits Science, Technology and Econo$m y$ (首届海峡两岸科技与经济论坛论文集). Fujian Science and Technology Press, Fuzhou, 183 - 188. (in Chinese) 